J. Lake Sci.(湖泊科学), 2018, 30(2): 509-518

DOI 10. 18307/2018. 0222

(c) 2018 by Journal of Lake Sciences

\title{
水文干旱多变量联合设计及水库影响评估
}

\author{
涂新军 ${ }^{1,2}$, 杜晓霞 $^{1}$, 杜奕良 $^{1}$, 陈晓宏 ${ }^{1}$, 黎 坤 $^{1}$ \\ (1:中山大学水资源与环境研究中心, 广州 510275) \\ (2:广东省华南地区水安全调控工程技术研究中心,广州 510275)
}

\begin{abstract}
摘 要: 基于东江流域博罗站月径流数据, 采用游程理论提取水文干旱事件. 选用 Meta-Gaussian Copula 函数, 统计模拟 水文干旱指标的多变量联合分布. 采用 Kendall 联合重现期和最大可能权函数,设计给定联合超越重现期的水文干旱指 标组合值, 并定量评估水库径流调节作用对水文干旱多变量联合特征的影响. 结果表明: 东江流域水文干旱历时、强度和 峰值的统计优选分布均为韦布尔分布. 干旱指标之间具有较高的正相关性, Meta-Gaussian Copula 能够很好地模拟水文干 旱指标两变量和三变量联合分布. 基于任意两个变量联合设计和三变量联合设计,干旱指标设计组合值位于同频位置附 近, 且同一个干旱指标设计值在不同变量组合之间差别较小. 水库径流调节作用对于缓解东江流域水文干旱效果明显, 同一组干旱指标的多变量联合超越重现期在水库影响下明显变大. 联合超越重现期越小, 水库对联合设计值的影响程度 越大. 根据目前水库运行模式, 若要满足河道内最小管理流量目标, 联合超越重现期 $10 \mathrm{a}$ 一遇的干旱历时、强度和峰值依 然达到了约 3.89 4.04月、7.20 7.97 亿 $\mathrm{m}^{3}$ 和 2.99 3.12 亿 $\mathrm{m}^{3}$.
\end{abstract}

关键词: 水文干旱;多变量联合设计;Kendall 重现期;最大可能权函数;水库影响;东江流域

\section{Multivariate joint design of hydrological drought and impact of water reservoirs}

\author{
TU Xinjun ${ }^{1,2}$, DU Xiaoxia ${ }^{1}$, DU Yiliang ${ }^{1}$, CHEN Xiaohong ${ }^{1} \&$ LI Kun ${ }^{1}$ \\ (1: Department of Water Resources and Environment, Sun Yat-sen University, Guangzhou 510275, P.R.China) \\ (2: Center of Water Security Engineering and Technology in Southern China of Guangdong, Guangzhou 510275, P.R. China)
}

Abstract: Using monthly streamflow data in Dongjiang Basin, South China, hydrological drought events were truncated on the basis of the run theory. The multivariate joint distributions of droughts were determined by using the function of Meta-Gaussian Copula. An improved method for multivariate design, the most-likely weight function, was applied to select design values of drought properties for the given Kendall joint return periods. Besides, impacts of water reservoirs in the joint design values of drought properties were quantified. Results demonstrate that the Weibull distribution fit the marginals of drought properties well. The dependences of drought properties were highly positive and the Meta-Gaussian Copula effectively modelled their bivariate and trivariate joint distributions. Despite of the bivariate or trivariate design, a set of design values of drought properties almost occurred in the identical cumulative frequencies with slight difference. The remarkable decrease in drought properties of the Dongjiang Basin was attributed to the regulation of water reservoirs. The exceeding joint return periods significantly increase for a set of drought properties under the impacts of water reservoirs. The shorter the joint return period was, the larger the change of the design values was. However, the duration, severity and peak of drought for the joint return period by 10 years still accounted for 3.89-4.04 months, 7.207.97 and 2.99-3.12 hundred-million $\mathrm{m}^{3}$, respectively, provided that minimum instream flow was satisfied for water demand in the Dongjiang Basin.

Keywords: Hydrological drought; multivariate joint design; Kendall joint return period; most-likely weight function; impact of water reservoirs; Dongjiang Basin

水文干旱事件通常基于设定的河川径流國值, 采用游程方法提取 ${ }^{[1-2]}$. 一次干旱事件一般包含历时、强 度和峰值等特性指标,因此水文干旱事件是多个变量 (两变量或三变量) 随机过程,且变量之间存在相依性.

* 国家自然科学基金项目 (51479217)资助. 2017-03-31 收稿; 2017-06-05 收修改稿. 涂新军(1972 ), 男,博士, 副 教授; E-mail: eestxj@ mail.sysu.edu.cn. 
Copula 是基于变量相依性的多变量边缘分布连接函数,已经广泛应用于水文干旱多变量联合分布模拟 ${ }^{[3-6]}$.

Copula 函数及边缘分布确定后, 传统联合重现期分析在判断安全或危险事件上有可能存在误判. Kendall 联合重现期概念的提出和应用, 改进了多变量联合设计的可靠性 ${ }^{[7-8]}$. 某一特定联合重现期是由无 穷多个变量组合值构成,如两变量的等值线和三变量的等值面. 如何在特定联合重现期的等值线或等值面 上, 选取一组设计值的联合分布设计研究逐渐受到关注. 传统设计方法是基于同频思路选取多变量设计组 合值 ${ }^{[9-10]}$, 但这种方法过于主观, 没有考虑边缘和联合分布的特性. Salvadori 等 ${ }^{[7]}$ 定义了最大可能权函数 (Most-likely weight function), 基于边缘及联合分布密度选取最大可能发生的多变量组合.

随着区域经济快速发展和流域水资源开发利用, 人类活动特别是大型水库的建设运行, 显著改变了河 川径流过程, 从而影响水文干旱特征. 目前, 定量评估水库径流调节作用对水文干旱的影响研究较少, 尹正 杰等 ${ }^{[11]}$ 定量刻画了水库径流调节对水文干旱单一指标的影响, 但没有考虑对水文干旱联合分布的影响.

因此, 本文以东江流域为例, 以满足当地需水要求的河道内最小流量管理目标为阈值, 基于水库人库和 出库流量还原河川天然径流过程, 采用游程理论分别提取实测和天然径流过程的水文干旱事件, 构建水文 干旱指标多变量联合分布 Copula 模型, 基于 Kendall 联合重现期并采用最大可能权函数设计多变量组合值, 通过对比实测和天然径流过程的水文干旱联合分布特征, 定量评估水库径流调节作用对水文干旱多变量联 合特征的影响程度, 为水文干旱多变量模拟和设计提供了一种新方法, 并为当地抗旱减灾的水资源多维风 险评估和管理提供科学依据.

\section{1 方法和数据}

\section{1 干旱指标联合分布与边缘分布模拟}

根据 Sklar 定理,多变量联合分布可定义为:

$$
F\left(x_{1}, \cdots, x_{d}\right)=C\left[F\left(x_{1}\right), \cdots, F\left(x_{d}\right)\right]=C\left(u_{1}, \cdots, u_{d}\right)
$$

式中, $C\left(u_{1}, \cdots, u_{d}\right)$ 为边缘分布在 $[0,1]$ 区间的 Copula 联结函数, $u_{1}=F\left(x_{1}\right), \cdots, u_{d}=F\left(x_{d}\right)$ 为各单变量的边 缘分布, 即累积频率. $d$ 为联合分布的维数, 即变量个数. 当模拟水文干旱指标三变量联合分布, $d=3, x_{1} 、 x_{2}$ 和 $x_{3}$ 分别为干旱特征的 3 个指标: 历时、强度和峰值; 当模拟干旱指标两变量联合分布时,则 $d=2, x_{1}$ 和 $x_{2}$ 为干旱指标中任意 2 个.

Copula 函数构造多样, 其中 Meta-Elliptic Copulas 的 Meta-Gaussian Copula 模型, 在水文干旱多变量联合 分布模拟中具有良好效果 ${ }^{[12]} . d$ 维 Meta-Gaussian Copula 的分布函数 $C\left(u_{1}, \cdots, u_{d}\right)$ 和密度函数 $c\left(u_{1}, \cdots, u_{d}\right)$ 分别为:

$$
\begin{gathered}
C\left(u_{1}, \cdots, u_{d}\right)=\int_{-\infty}^{b_{1}} \cdots \int_{-\infty}^{b_{d}} g\left(\omega_{1}, \cdots, \omega_{d}\right) d \omega_{1} \cdots d \omega_{d} \\
c\left(u_{1}, \cdots, u_{d}\right)=|\Sigma|^{-1 / 2} \exp \left(-\zeta^{T} \Sigma^{-1} \zeta / 2+\zeta^{T} \zeta / 2\right)
\end{gathered}
$$

其中:

$$
g\left(\omega_{1}, \cdots, \omega_{d}\right)=(2 \pi)^{-d / 2}|\Sigma|^{-1 / 2} \exp \left(-\omega^{T} \Sigma^{-1} \omega / 2\right)
$$

式中, $b_{1}=\Phi^{-1}\left(u_{1}\right), \cdots, b_{d}=\Phi^{-1}\left(u_{d}\right)$, 其中 $\Phi^{-1}(\cdot)$ 为标准正态分布的逆函数; $\Sigma$ 为相关系数矩阵是模型参 数, $\omega$ 为被积函数变量矩阵 $\omega=\left[\omega_{1}, \cdots, \omega_{d}\right]^{T}, \zeta=\left[b_{1}, \cdots, b_{d}\right]^{T}$.

变量间相关性采用 Kendall $\tau$ 、Spearman 秩相关系数 $\rho$ 和 Pearson 古典相关系数 $\gamma$, 联合分布模型拟合检 验采用基于 Rosenblatt 变换的 Kolmogorov-Smirnov ( K-S ) 法 ${ }^{[13]}$. 边缘分布考虑三参数的广义极值分布 (GEV)、皮尔逊 III 型 (P-III)、广义帕累托分布 (GP) 和两参数的韦布尔分布 (WBL)、对数正态分布 (LOGN)、伽马分布 (GAMA) 共 6 种常用模型, 通过 K-S 法和 Anderson-Darling (A-D) 法进行拟合检验, 并综 合考虑均方根误差 $(R M S E)$ 、赤池信息准则 $(A I C)$ 和检验统计量概率 $P$ 值进行优选 ${ }^{[14-15]}$. 联合分布及边缘分 布模型参数均采用最大似然法估计.

\section{2 超越概率、重现期与设计值}

就水文干旱特征研究来说, 通常关注干旱指标大于设定阈值的概率或重现期,即:

$$
\hat{P}=1-F(x)=1-u=\hat{u}
$$




$$
\hat{T}=1 /\left(N_{E} \cdot \hat{P}\right)=1 /\left(N_{E} \cdot \hat{u}\right)
$$

式中, $F(x)$ 或 $u$ 为历时、强度或峰值的累积频率, $\hat{P}$ 或 $\hat{u}$ 为超越概率, $\hat{T}$ 为超越重现期, $N_{E}$ 为干旱事件年均 发生次数.

干旱指标两变量联合超越概率 $\hat{C}$ 为:

$$
\hat{C}=1-\left(u_{1}+u_{2}\right)+C\left(u_{1}, u_{2}\right)
$$

式中, $\left(u_{1}, u_{2}\right)$ 为历时、强度和峰值其中任意两变量组合.

干旱指标三变量联合超越概率 $\hat{C}$ 为:

$$
\hat{C}=1-\left(u_{1}+u_{2}+u_{3}\right)+C\left(u_{1}, u_{2}\right)+C\left(u_{1}, u_{3}\right)+C\left(u_{2}, u_{3}\right)-C\left(u_{1}, u_{2}, u_{3}\right)
$$

式中, $\left(u_{1}, u_{2}, u_{3}\right)$ 为历时、强度和峰值的三变量组合.

Kendall 联合重现期是以联合概率相等的一系列事件为临界曲线, 来识别危险事件或安全事件 ${ }^{[7-8]}$. 因 此,干旱指标的 Kendall 联合重现期, 其对应的联合超越概率可用 Kendall 分布函数 $K_{\hat{C}}$ 来表示, 即

$$
K_{\hat{C}}=P(\hat{C} \leqslant q)
$$

式中, $q \in(0,1), \hat{C}$ 为干旱指标两变量或三变量联合超越概率, $P$ 为 $\hat{C} \leqslant q$ 的分布概率.

由于 $K_{C}$ 无法通过解析式求解, 一般是在联合分布模型确定的基础上, 采用 Monte Carlo 法模拟生成组 合,通过经验频率公式估算, 即:

$$
K_{\hat{C}}=\frac{1}{n} \sum_{i=1}^{n} 1(\hat{C} \leqslant q)
$$

式中, $n$ 为 Monte Carlo 模拟组合的数目,通常可取 $10^{4}$ 或更大.

由此可以进一步计算干旱指标两变量或三变量的 Kendall 联合超越重现期 $\hat{T}_{K}$, 即:

$$
\hat{T}_{K}=1 /\left(N_{E} \cdot K_{\hat{C}}\right)
$$

单变量的重现期和设计值是一一对应的. 但是对于多变量来说, 任一给定的联合重现期理论上是有无 数种组合值. 但在水文水资源工程与管理实践中, 有时需要明确给出一种设计组合. 为此, 可定义最大可能 权函数 ${ }^{[7]}$, 即:

$$
\begin{gathered}
\left.\left(u_{1, m}, \cdots, u_{d, m}\right)\right|_{\hat{r}_{k}}=\arg \max f\left(x_{1}, \cdots, x_{d}\right) \\
f\left(x_{1}, \cdots, x_{d}\right)=c\left(u_{1}, \cdots, u_{d}\right) f\left(x_{1}\right), \cdots, f\left(x_{d}\right)
\end{gathered}
$$

式中, $\left(u_{1, m}, \cdots, u_{d, m}\right)$ 为该方法选取的累积频率组合, 反映了给定联合重现期 $\hat{T}_{K}$ 最大可能出现的一种组合, $f\left(x_{1}, \cdots, x_{d}\right)$ 为给定联合重现期 $\hat{T}_{K}$ 所有组合的联合分布概率密度 $c\left(u_{1}, \cdots, u_{d}\right)$ 和各个边缘分布概率密度 $f\left(x_{1}\right), \cdots, f\left(x_{d}\right)$ 的积.

具体设计步骤如下: 1) 基于干旱指标样本值确定的边缘及联合分布模型和参数,然后采用 Monte Carlo 法模拟 $m$ 次 $n$ 组 $\left(u_{1}, u_{2}\right)$ 或 $\left(u_{1}, u_{2}, u_{3}\right), m$ 和 $n$ 均取 $10^{4}$ 或更大;2) 根据式 $(5) \sim(11)$ 可反算出 $m$ 组任一给 定联合重现期 $\hat{T}_{K}$ 的 $\left(u_{1, \hat{T}_{k}}, u_{2, \hat{T}_{k}}\right)$ 或 $\left(u_{1, \hat{T}_{K}}, u_{2, \hat{r}_{k}}, u_{3, \hat{T}_{n}}\right)$, 依据公式 $(12) \sim(13)$ 选出 $\left(u_{1, m}, u_{2, m}\right)$ 或 $\left(u_{1, m}, u_{2, m}\right.$, $\left.\left.u_{3, m}\right) ; 3\right)$ 相应地干旱指标设计组合值可根据各自边缘分布的反函数计算得出, 即:

\section{3 水库径流调节对水文干旱的影响}

$$
x_{1}=F^{-1}\left(u_{1, m}\right), x_{2}=F^{-1}\left(u_{2, m}\right), x_{3}=F^{-1}\left(u_{3, m}\right)
$$

为了定量评估水库径流调节对水文干旱的影响, 可定义相对变化 $R_{v}$ :

$$
R_{v}=100 \cdot\left(D_{1}-D_{2}\right) / D_{2}
$$

式中, $R_{v}$ 为实测径流的水文干旱特征指标相对于天然径流的变化 (\%) , 即水库径流调节对水文干旱特征变 化的影响程度; $D_{1}$ 和 $D_{2}$ 分别为实测径流和天然径流的水文干旱特征指标或设计值.

\section{4 流域概况及数据}

东江流域属于中国珠江水系 (图 1), 流域控制站博罗以上集水面积分别为 $25325 \mathrm{~km}^{2}$. 东江流域水资源 开发程度较高,新丰江水库、枫树坝水库和白盆珠水库三大水库分别运行于 1961、1974 和 1986 年,均为多年 调节型水库, 对流域河川径流过程影响显著 ${ }^{[16-17]}$. 随着区域经济的快速发展, 东江流域近些年的水资源供需 矛盾日益突显, 特别是 2004-2005 年的冬、春季极端干旱, 严重威胁到了东江流域下游及珠江三角洲部分地 
区的供水安全. 为此, 《广东省东江流域水资源分配方案》规定了东江干流重要断面的最小流量管理目标, 其 中博罗站流量为 $320 \mathrm{~m}^{3} / \mathrm{s}$.

本文基于博罗站 1954 年 4 月- 2009 年 3 月共 $55 \mathrm{a}$ (水文年) 的实测月径流过程, 根据三大水库人库和 出库的月平均流量, 计算水库调节的月径流量 (出库一人库). 实测月径流过程扣除水库调节的月径流量, 形 成了博罗站天然径流系列. 自新丰江水库运行起至今的实测径流系列 (1961 年 4 月- 2009 年 3 月), 实际上 反映了东江水文干旱受到流域水库的径流调节影响. 以最小管理流量 $320 \mathrm{~m}^{3} / \mathrm{s}$ 为阈值, 根据游程方法分别 截取实测系列和天然系列的水文干旱事件, 每一次干旱事件的干旱指标包括历时、强度和峰值, 分别反映干 旱持续时间、干旱期间的总缺水量和最大的月缺水量.

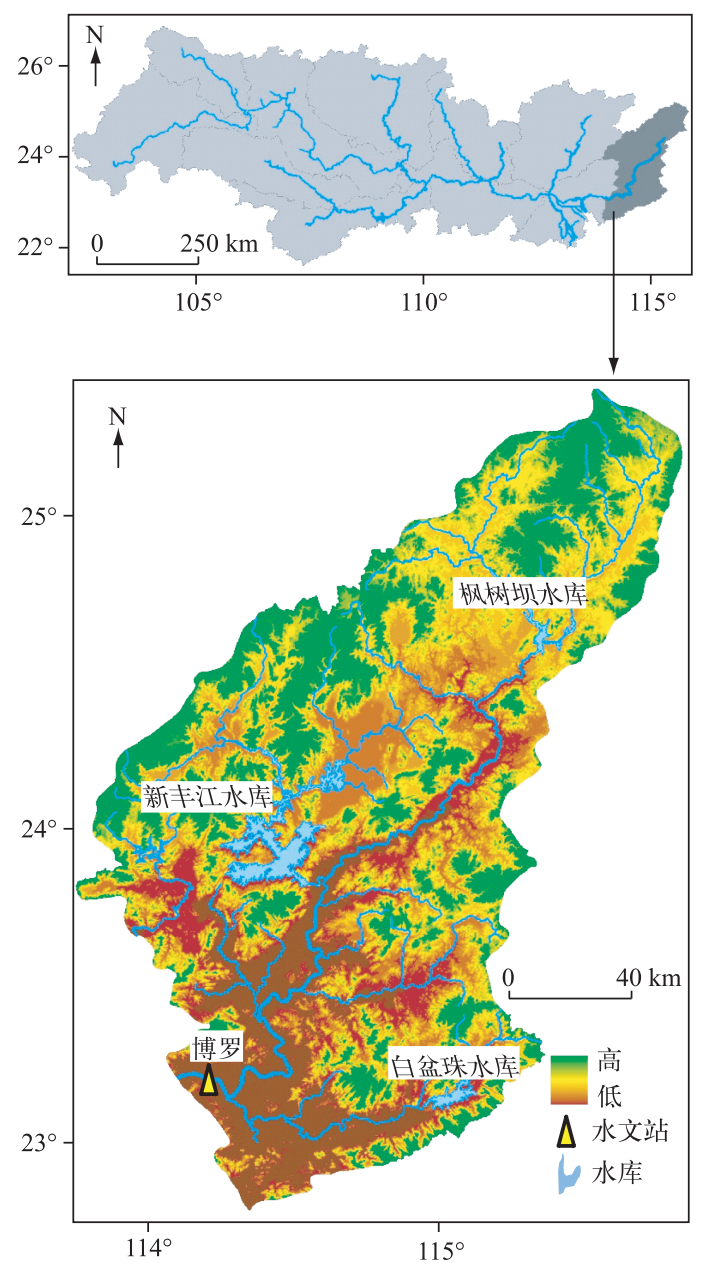

图 1 东江流域地理位置、水文站点及三大水库分布

Fig.1 Location of Dongjiang Basin, the hydrological gauging station and distribution of three reservoirs

\section{2 结果与讨论}

\section{1 水文干旱特征}

图 2 为东江流域博罗站的月径流过程 (纵坐标采用了对数刻度), 低于阈值的部分即为发生的水文干旱 事件. 1954-2009 年期间, 还原的天然径流水文干旱事件次数为 58 次, 年均约 1.05 次. 经过水库径流调节 作用的 1961-2009 年期间,干旱事件实际发生 30 次,年均约 0.63 次,减少了约 40.7\%. 


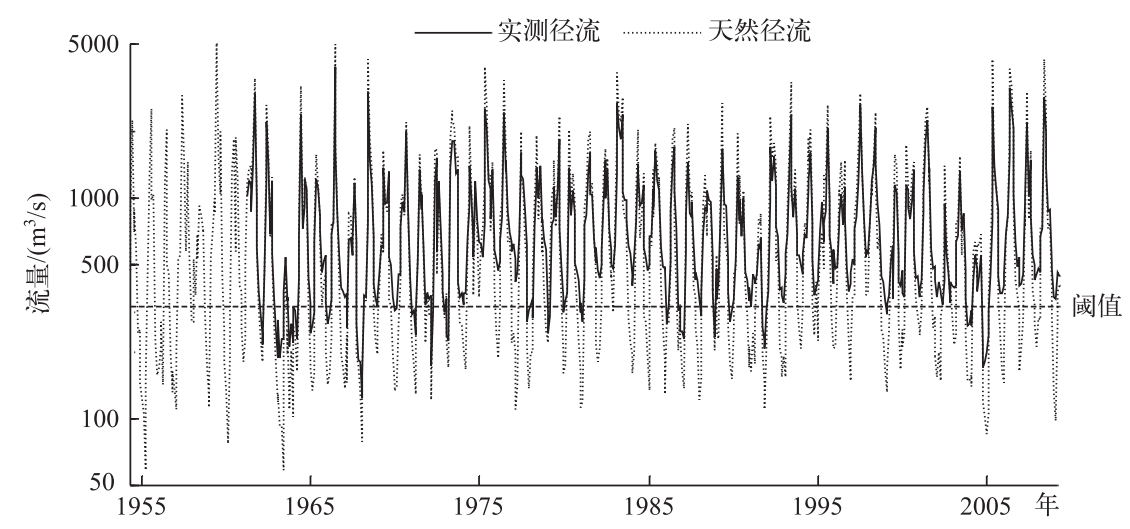

图 2 月径流过程及水文干旱事件

Fig. 2 Streamflow processes and hydrological drought events

干旱指标统计特征见表 1. 天然径流的干旱事件最长历时为 8 个月, 分别出现在 1962 年 11 月-1963 年 6 月和 1963 年 8 月一 1964 年 3 月,历时达到 7 个月分别出现在 1954 年 10 月一 1955 年 4 月、1 955 年 10 月一 1956 年 4 月、 1990 年 10 月－1991 年 4 月、1998 年 10 月- 1999 年 4 月和 2001 年 11 月 - 2002 年 5 月. 在水 库径流调节作用下, 干旱事件最长历时为 6 个月, 分别发生在 1962 年 12 月- 1963 年 5 月和 2004 年 10 月一 2005 年 3 月.

干旱最大强度的总缺水量约为 35.12 亿 $\mathrm{m}^{3}$, 出现在 1962 年 11 月- 1963 年 6 月. 最大峰值的月缺水量 约为 6.80 亿 $\mathrm{m}^{3}$,出现在 1955 年 3 月和 1963 年 5 月,博罗站流量约为 $57.7 \mathrm{~m}^{3} / \mathrm{s}$. 经过水库径流调节作用,最 大强度的总缺水量约为 17.82 亿 $\mathrm{m}^{3}$, 发生在 1967 年 10 月- 1968 年 2 月. 最大峰值的月缺水量约为 5.06 亿 $\mathrm{m}^{3}$, 发生在 1968 年 1 月,博罗站流量约为 $124.6 \mathrm{~m}^{3} / \mathrm{s}$.

水库径流调节作用通常会缩短干旱历时, 并降低干旱强度. 历时、强度和峰值的均值分别由 4.07 月、 12.63 亿 $\mathrm{m}^{3}$ 和 4.15 亿 $\mathrm{m}^{3}$, 降低到 2.37 月、3.91 亿 $\mathrm{m}^{3}$ 和 1.81 亿 $\mathrm{m}^{3}$, 分别减少了 $41.8 \% 、 69.0 \%$ 和 $56.5 \%$; 中位 值分别由 4.00 月、 12.19 亿 $\mathrm{m}^{3}$ 和 4.41 亿 $\mathrm{m}^{3}$, 降低到 2.00 月、 2.11 亿 $\mathrm{m}^{3}$ 和 1.56 亿 $\mathrm{m}^{3}$, 分别减少了 $50.0 \%$ 、 $82.7 \%$ 和 $64.7 \%$. 最小 1 个月的干旱强度由 0.46 亿 $\mathrm{m}^{3}$ 降低到 0.03 亿 $\mathrm{m}^{3}$,减少了 $93.3 \%$ (表 1).

表 1 干旱指标统计特征

Tab.1 Statistical characteristics of drought properties

\begin{tabular}{|c|c|c|c|c|c|c|c|c|c|}
\hline \multirow{2}{*}{ 统计特征 } & \multicolumn{3}{|c|}{ 实测径流 } & \multicolumn{3}{|c|}{ 天然径流 } & \multicolumn{3}{|c|}{ 相对变化/\% } \\
\hline & 历时/月 & 强度/亿 $\mathrm{m}^{3}$ & 峰值/亿 $\mathrm{m}^{3}$ & 历时/月 & 强度/亿 $\mathrm{m}^{3}$ & 峰值/亿 m ${ }^{3}$ & 历时 & 强度 & 峰值 \\
\hline 最大值 & 6.00 & 17.82 & 5.06 & 8.00 & 35.12 & 6.80 & -25.0 & -49.2 & -25.5 \\
\hline 均值 & 2.37 & 3.91 & 1.81 & 4.07 & 12.63 & 4.15 & -41.8 & -69.0 & -56.5 \\
\hline 中位值 & 2.00 & 2.11 & 1.56 & 4.00 & 12.19 & 4.41 & -50.0 & -82.7 & -64.7 \\
\hline 最小值 & 1.00 & 0.03 & 0.03 & 1.00 & 0.46 & 0.46 & 0 & -93.3 & -93.3 \\
\hline
\end{tabular}

\section{2 干旱指标边缘分布特征及设计值}

干旱指标边缘分布拟合检验结果显示, 历时只接受 WBL 模型, 强度和峰值能接受除了 GP 模型之外的 其他备选模型. 综合考虑 $R M S E 、 A I C$ 和检验统计量概率 $P$ 值的计算结果, 实测和天然径流的干旱指标边缘 分布均采用 WBL 模型, 其 $P$ 值均大于显著性检验水平 0.05 , 见表 2. 经验和理论的累积频率 $Q-Q$ 关系图显 示 (图 3), 频率关系点据基本上在等值线附近, 强度和峰值呈现出更好的拟合效果. WBL 模型的累积频率分 布 $F(x)$ 和密度函数 $f(x)$ 分别为:

$$
F(x)=1-\mathrm{e}^{-(x / \lambda)^{k}}
$$


式中, $k$ 和 $\lambda$ 为模型参数.

$$
f(x)=(k / \lambda)(x / \lambda)^{k-1} \mathrm{e}^{-(x / \lambda)^{k}}
$$

由于水库径流调节作用改变了东江水文干旱特征, 尽管对于同一个干旱指标来说, 实测径流和天然径 流的最优分布模型相同,但二者之间的参数差别明显 (表 2).

表 2 干旱指标边缘分布

Tab.2 Marginal distribution of drought properties

\begin{tabular}{|c|c|c|c|c|c|c|}
\hline \multirow{2}{*}{ 干旱指标 } & \multicolumn{3}{|c|}{ 实测径流 } & \multicolumn{3}{|c|}{ 天然径流 } \\
\hline & 分布模型 & $P$ 值 & 模型参数 & 分布模型 & $P$ 值 & 模型参数 \\
\hline 历时 & WBL & 0.187 & $k=2.674, \lambda=1.704$ & WBL & 0.267 & $k=4.585, \lambda=2.434$ \\
\hline 强度 & WBL & 0.952 & $k=3.682, \lambda=0.890$ & WBL & 0.988 & $k=14.065, \lambda=1.638$ \\
\hline 峰值 & WBL & 0.956 & $k=1.976, \lambda=1.423$ & WBL & 0.645 & $k=4.611, \lambda=3.371$ \\
\hline
\end{tabular}

(a)历时

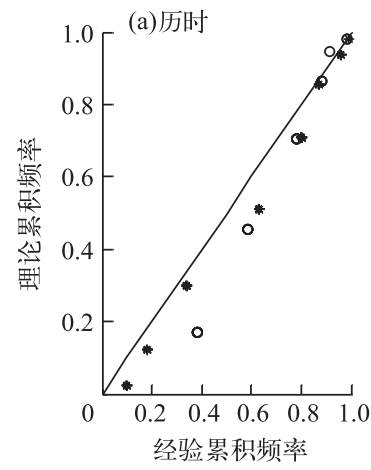

实测径流 $*$ 天然径流

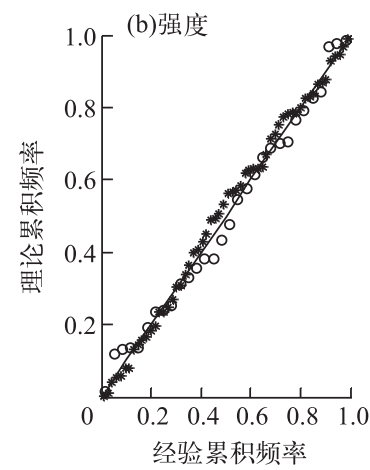

(c)峰值

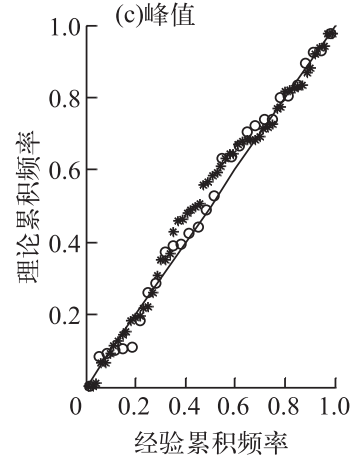

图 3 干旱指标边缘分布经验与理论累积频率 $Q-Q$ 关系

Fig.3 $Q-Q$ of empirical and theoretical cumulative frequencies of marginal distribution of drought properties

根据干旱指标边缘理论分布和参数, 各单变量重现期设计值变化如图 4(横坐标采用对数坐标). 与天然 径流相比较, 实测径流的设计值变化曲线向下移动, 这表明在水库径流调节作用下, 干旱指标单变量设计值明 显变小. 重现期介于 2 100 a 之间,历时、强度和峰值分别下降了约 2.48 2.96 月、11.08 17.83亿 $\mathrm{m}^{3}$ 和 1.92 3.54 亿 $\mathrm{m}^{3}$. 随着重现期增大, 水库的影响程度 (相对变化的绝对值) 逐渐减少. 历时、强度和峰值 $2 \mathrm{a}$ 一遇设计 值的下降幅度分别约为 $72.7 \%$ 、94.2\% 和 $83.7 \%, 100 \mathrm{a}$ 一遇设计值则分别降低到约为 $28.7 \%$ 、 $49.5 \%$ 和 $26.4 \%$. 主 要原因是水库径流调节作用增加了下游干旱期间的径流量, 且对低重现期的干旱指标影响程度更大.

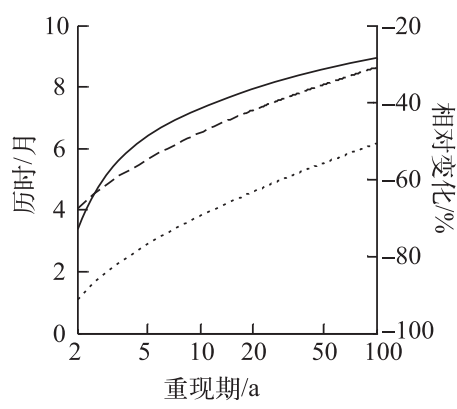

……实测径流 - - - 天然径流 — 相对变化
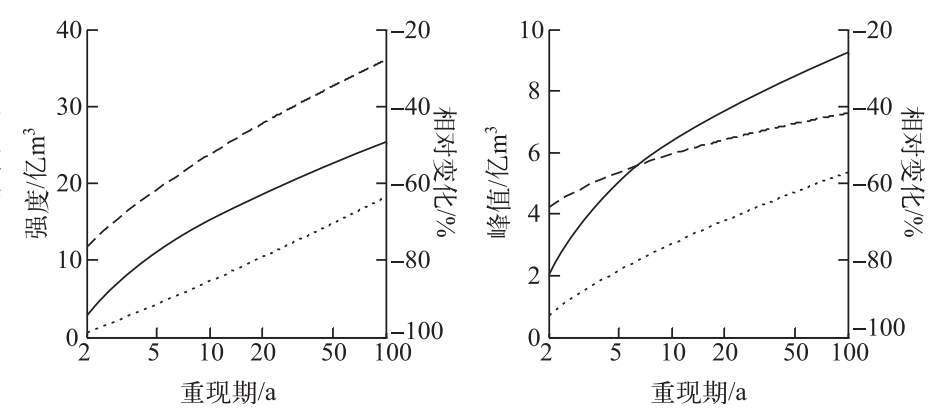

图 4 干旱指标单变量重现期设计值

Fig.4 Change of design value of the univariate return period for drought properties 


\section{3 干旱指标联合分布特征及设计值}

经过水库径流调节作用,历时一强度的相关系数 略有下降, 而历时一峰值和强度一峰值的相关系数 均有所上升. 干旱指标之间的 Kendall、Spearman 和 Pearson 相关系数均大于 0.5 , 表明东江水文干旱指标 之间具有较高的正相关性 (表 3 ).

联合分布经验和理论的累积频率 $Q-Q$ 关系显 示 (图 5), 频率关系点据基本上在等值线附近. 但与 历时有关联合分布拟合图中 (图 5a、5b 和 5d), 低值 部分点据向下偏离等值线,即理论频率明显大于经验频率,其主要原因是干旱历时在低值部分的重复值较 多. 拟合检验结果显示, 检验统计量的概率 $P$ 值均明显大于显著性检验水平 0.05 (表 4), 表明 Meta-Gaussian Copula 能够很好地模拟东江流域水文干旱指标两变量和三变量联合分布. 干旱指标两变量联合分布参数见 表 4 , 而三变量联合分布参数是由两两分布参数构成的矩阵.

(a) 历时一强度

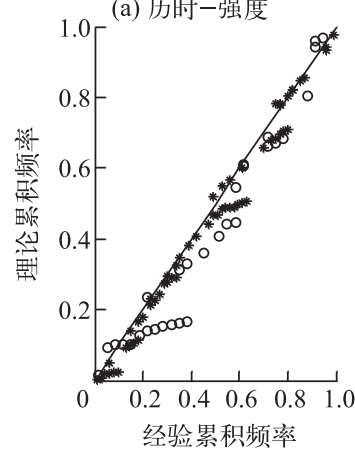

(b) 历时一峰值

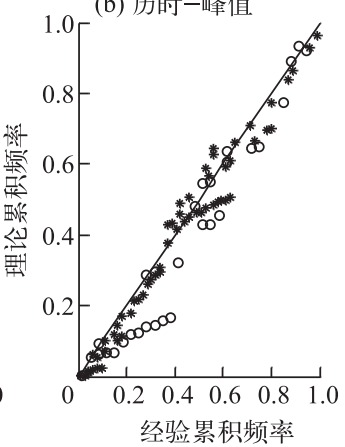

* 天然径流

(c) 强度一峰值

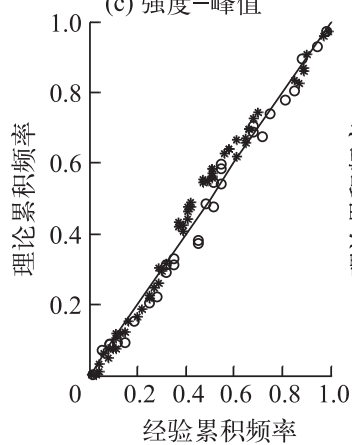

(d) 历时一强度一峰值

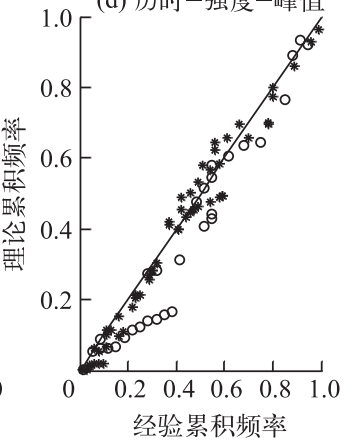

图 5 干旱指标联合分布经验与理论累积频率 $Q-Q$ 关系

Fig.5 $Q-Q$ of empirical and theoretical cumulative frequencies of joint distribution of drought properties

表 4 干旱指标联合分布

Tab.4 Joint distribution of drought properties

\begin{tabular}{|c|c|c|c|c|c|c|}
\hline \multirow{2}{*}{ 指标组合 } & \multicolumn{3}{|c|}{ 实测径流 } & \multicolumn{3}{|c|}{ 天然径流 } \\
\hline & 分布模型 & $P$ 值 & 模型参数 & 分布模型 & $P$ 值 & 模型参数 \\
\hline 历时一强度 & Meta-Gaussian & 0.535 & 0.863 & Meta-Gaussian & 0.878 & 0.908 \\
\hline 历时一峰值 & Meta-Gaussian & 0.809 & 0.689 & Meta-Gaussian & 0.789 & 0.725 \\
\hline 强度一峰值 & Meta-Gaussian & 0.678 & 0.941 & Meta-Gaussian & 0.709 & 0.897 \\
\hline 历时一强度一峰值 & Meta-Gaussian & 0.775 & & Meta-Gaussian & 0.695 & \\
\hline
\end{tabular}

基于干旱指标联合分布模型和参数,干旱指标两变量联合超越重现期为 $2 、 5 、 10 、 20 、 50$ 和 $100 \mathrm{a}$ 的等值 散点线如图 6(双坐标轴均采用海森坐标). 与天然径流相比较, 实测径流的干旱指标联合超越重现期等值 散点线向坐标原点内移, 表明任意一对干旱指标, 其两变量联合超越重现期在流域水库径流调节作用后明 显变大. 基于最大权函数设计的两变量累积频率组合如图 6 中的空心点位置, 结果显示干旱指标的一对组 合值基本位于同频位置 (图 6 中的对角线) 附近, 即 $u_{1, m} \approx u_{2, m}$.

干旱指标三变量联合超越重现期等值散点面如图 7, 变化特征与两变量类似. 与天然径流相比较, 实测 径流的干旱指标联合超越重现期等值散点面向坐标原点内移, 表明同一组干旱指标, 其三变量联合超越重 现期在流域水库径流调节作用后明显变大. 尽管三变量联合超越重现期等值散点面在二维平面投影的两变 
(a)历时一强度

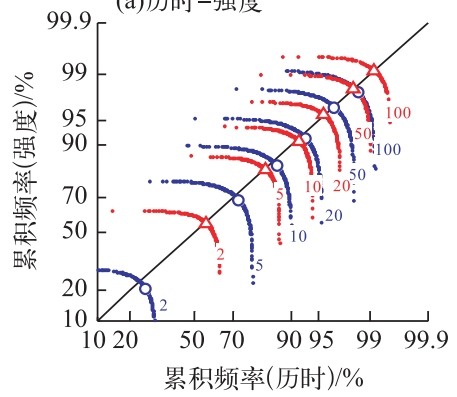

○实测径流 $\Delta$ 天然径流

(b)历时一峰值

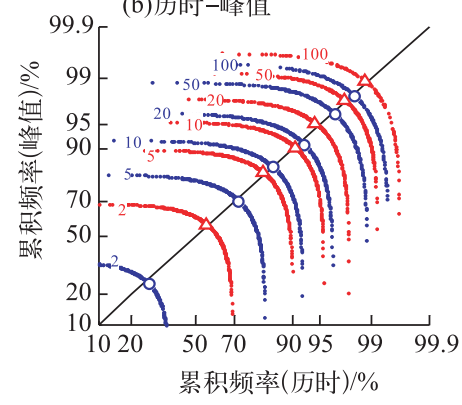

(c)强度一峰值

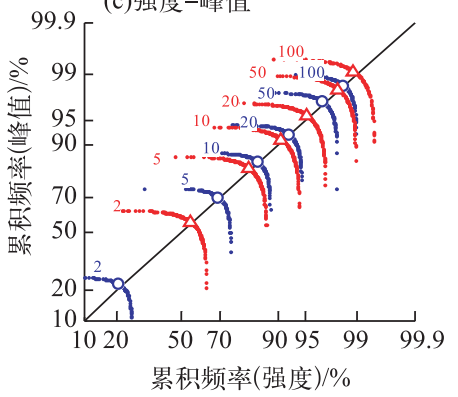

图 6 干早指标两变量联合超越重现期

Fig.6 Bivariate exceeding joint return period of drought properties

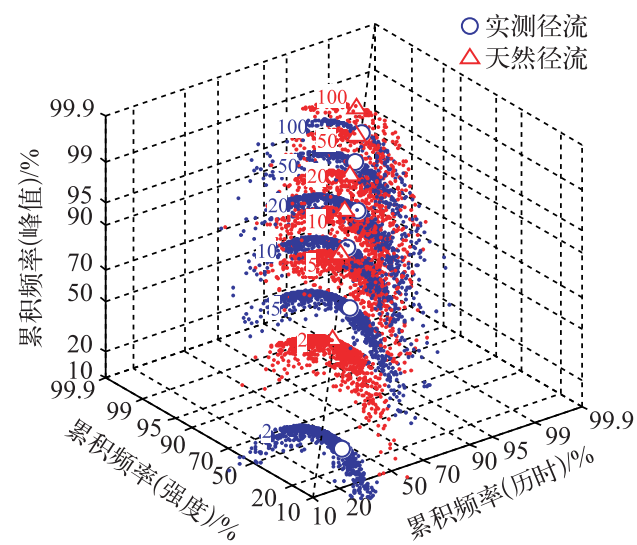

图 7 干早指标三变量联合超越重现期

Fig.7 Trivariate exceeding joint return period of drought properties
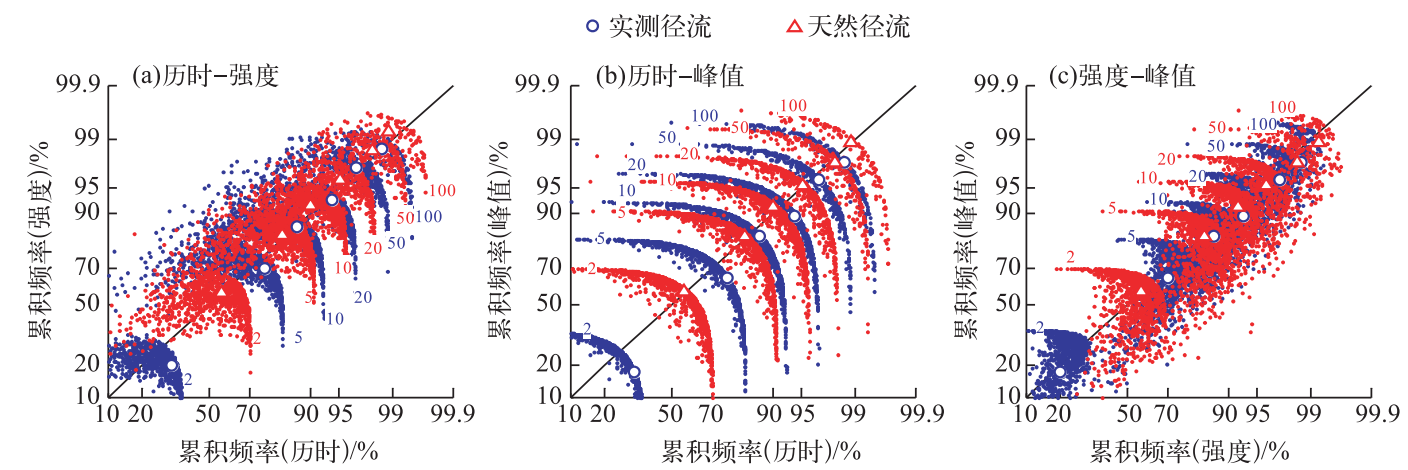

图 8 干旱指标三变量联合超越重现期的二维投影

Fig. 8 Two-dimensional projection of trivariate exceeding joint return period of drought properties

量组合点比较分散 (图 8), 但最大可能权函数设计的干旱指标三变量组合, 也基本上处于同频位置( 图 7 和 图 8 中的对角线) 附近, 即 $u_{1, m} \approx u_{2, m} \approx u_{3, m}$. 
干旱指标联合超越重现期的设计组合值结果见表 5. 不管是任意两个变量的联合设计,还是三变量联合 设计, 单个干旱指标设计值差别较小. 以联合超越重现期 $10 \mathrm{a}$ 一遇为例, 天然径流的设计干旱历时、强度和 峰值分别约为 7.07 7.34 月、23.92 24.77 亿 $\mathrm{m}^{3}$ 和 5.94 6.04 亿 $\mathrm{m}^{3}$, 实测径流的则分别降低到约为 3.89 4.04 月、7.20 7.97 亿 $\mathrm{m}^{3}$ 和 $2.99 \sim 3.12$ 亿 $\mathrm{m}^{3}$.

表 5 干旱指标联合设计值

Tab.5 Joint design values of drought properties

\begin{tabular}{|c|c|c|c|c|c|c|c|c|c|c|c|c|c|}
\hline \multirow{2}{*}{ 指标组合 } & \multirow{2}{*}{ 干旱指标 } & \multicolumn{6}{|c|}{ 实测径流 } & \multicolumn{6}{|c|}{ 天然径流 } \\
\hline & & $2 \mathrm{a}$ & $5 \mathrm{a}$ & $10 \mathrm{a}$ & $20 \mathrm{a}$ & $50 \mathrm{a}$ & $100 \mathrm{a}$ & $2 \mathrm{a}$ & $5 \mathrm{a}$ & $10 \mathrm{a}$ & $20 \mathrm{a}$ & $50 \mathrm{a}$ & $100 \mathrm{a}$ \\
\hline \multirow[t]{2}{*}{ 历时一强度 } & 历时/月 & 1.33 & 3.09 & 4.01 & 4.75 & 5.54 & 6.21 & 4.26 & 5.80 & 6.67 & 7.34 & 8.16 & 8.70 \\
\hline & 强度/亿 m ${ }^{3}$ & 0.70 & 4.37 & 7.20 & 10.26 & 14.70 & 17.26 & 12.38 & 19.66 & 24.16 & 28.57 & 33.13 & 36.53 \\
\hline \multirow[t]{2}{*}{ 历时一峰值 } & 历时/月 & 1.35 & 3.07 & 3.89 & 4.68 & 5.53 & 6.07 & 4.23 & 5.70 & 6.54 & 7.07 & 7.87 & 8.43 \\
\hline & 峰值/亿 m ${ }^{3}$ & 0.81 & 2.25 & 3.07 & 3.69 & 4.58 & 5.18 & 4.40 & 5.45 & 5.94 & 6.42 & 6.85 & 7.20 \\
\hline \multirow[t]{2}{*}{ 强度一峰值 } & 强度/亿 $\mathrm{m}^{3}$ & 0.70 & 4.39 & 7.39 & 10.65 & 15.02 & 18.23 & 12.32 & 19.41 & 23.92 & 27.72 & 32.63 & 35.18 \\
\hline & 峰值/亿 m³ & 0.76 & 2.25 & 3.12 & 3.87 & 4.87 & 5.40 & 4.36 & 5.46 & 6.02 & 6.48 & 6.98 & 7.30 \\
\hline \multirow[t]{3}{*}{ 历时一强度一峰值 } & 历时/月 & 1.52 & 3.29 & 4.04 & 4.92 & 5.57 & 6.26 & 4.24 & 5.74 & 6.48 & 7.22 & 8.07 & 8.50 \\
\hline & 强度/亿 $\mathrm{m}^{3}$ & 0.68 & 4.51 & 7.97 & 11.02 & 15.65 & 18.89 & 12.70 & 20.30 & 24.77 & 28.90 & 33.75 & 37.07 \\
\hline & 峰值/亿 m³ & 0.62 & 2.03 & 2.99 & 3.51 & 4.51 & 5.03 & 4.42 & 5.49 & 6.04 & 6.43 & 6.85 & 7.21 \\
\hline
\end{tabular}

很显然, 实测径流的各干旱指标设计值均明显小于天然径流, 表明东江流域的水库径流调节作用对于 缓解当地水文干旱效果明显. 联合超越重现期越小, 水库径流调节对干旱指标设计值的影响程度 (设计值相 对变化的绝对值) 越大. 如 $100 \mathrm{a}$ 一遇的联合超越重现期, 其干旱历时、强度和峰值设计值的相对变化绝对 值, 分别约为 $26.3 \% \sim 28.6 \% 、 48.2 \% \sim 52.7 \%$ 和 $26.1 \% \sim 30.2 \%$; 联合重现期 $2 \mathrm{a}$ 一遇, 历时、强度和峰值的相对 变化程度 (绝对值) 分别上升为 $64.0 \% \sim 68.8 \% 、 94.3 \% \sim 94.6 \%$ 和 $81.6 \% \sim 86.1 \%$ (表 6 ). 影响程度从大到小依 次为强度、峰值和历时.

表 6 干旱指标设计值的相对变化 $(\%)$

Tab.6 Change in joint design values of drought properties

\begin{tabular}{|c|c|c|c|c|c|c|c|c|c|}
\hline \multirow{2}{*}{$\begin{array}{c}\text { 联合 } \\
\text { 重现期 }\end{array}$} & \multicolumn{2}{|c|}{ 历时一强度 } & \multicolumn{2}{|c|}{ 历时一峰值 } & \multicolumn{2}{|c|}{ 强度一峰值 } & \multicolumn{3}{|c|}{ 历时一强度一峰值 } \\
\hline & 历时 & 强度 & 历时 & 峰值 & 强度 & 峰值 & 历时 & 强度 & 峰值 \\
\hline $2 a$ & -68.8 & -94.3 & -68.0 & -81.6 & -94.3 & -82.5 & -64.0 & -94.6 & -86.1 \\
\hline $5 \mathrm{a}$ & -46.7 & -77.8 & -46.1 & -58.7 & -77.4 & -58.8 & -42.6 & -77.8 & -63.0 \\
\hline $10 \mathrm{a}$ & -39.9 & -70.2 & -40.6 & -48.4 & -69.1 & -48.3 & -37.7 & -67.8 & -50.4 \\
\hline $20 \mathrm{a}$ & -35.2 & -64.1 & -33.8 & -42.5 & -61.6 & -40.4 & -31.9 & -61.9 & -45.4 \\
\hline $50 \mathrm{a}$ & -32.1 & -55.6 & -29.7 & -33.2 & -54.0 & -30.2 & -31.0 & -53.6 & -34.1 \\
\hline $100 \mathrm{a}$ & -28.6 & -52.7 & -28.0 & -28.1 & -48.2 & -26.1 & -26.3 & -49.0 & -30.2 \\
\hline
\end{tabular}

\section{3 结论}

东江流域水文干旱历时、强度和峰值的统计最优分布均为 WBL 分布. 在水库径流调节作用下,干旱指 标单变量设计值明显变小. 随着重现期增大, 水库影响程度逐渐减少. 历时、强度和峰值 $2 \mathrm{a}$ 一遇设计值的下 降幅度分别约为 $72.7 \% 、 94.2 \%$ 和 $83.7 \%, 100 \mathrm{a}$ 一遇则分别降低到约为 $28.7 \% 、 49.5 \%$ 和 $26.4 \%$.

水文干旱指标之间具有较高的正相关性,Meta-Gaussian Copula 能够很好地模拟水文干旱指标两变量和 三变量联合分布. 同一组干旱指标,其两变量和三变量联合超越重现期在水库影响下明显变大. 基于最大可 能权函数设计结果显示, 水文干旱指标的两变量和三变量设计组合值基本均位于同频位置附近. 基于任意 两个变量的联合设计和三变量联合设计,单个干旱指标设计值差别较小. 联合超越重现期越小, 水库对联合 
设计值的影响程度越大.

在水库影响下, 联合超越重现期 $100 \mathrm{a}$ 一遇的历时、强度和峰值设计值分别下降了约 $26.3 \% \sim 28.6 \%$ 、 $48.2 \% \sim 52.7 \%$ 和 $26.1 \% \sim 30.2 \% ; 2 \mathrm{a}$ 一遇的分别下降了约 $64.0 \% \sim 68.8 \% 、 94.3 \% \sim 94.6 \%$ 和 $81.6 \% \sim 86.1 \%$. 受影响程度从大到小依次为强度、峰值和历时.

值得注意的是, 根据目前水库运行模式, 若要满足河道内最小管理流量目标, 联合超越重现期 $10 \mathrm{a}$ 一遇 的历时、强度和峰值依然达到了约 3.89 4.04 月、7.20 7.97 亿 $\mathrm{m}^{3}$ 和 $2.99 \sim 3.12$ 亿 $\mathrm{m}^{3}$.

\section{4 参考文献}

[ 1 ] Zelenhasić E, Salvai A. A method of streamflow drought analysis. Water Resources Research, 1987, 23 (1): 156-168. DOI: $10.1029 /$ WR023i001p00156.

[ 2 ] Tallaksen LM, Massen H, Clausen B. On the definition and modelling of streamflow drought duration and deficit volume. Hydrological Science Journal, 1997, 42(1) : 15-33. DOI: 10.1080/02626669709492003.

[ 3 ] Dong QJ, Xie P. Advances in hydrological drought research. Journal of China Hydrology, 2014, 34(4): 1-7. [董前进, 谢平. 水文干旱研究进展. 水文, 2014, 34(4): 1-7.]

[ 4 ] Cheng L, Jin JL, Li JQ et al. Advances in the study of drought frequency analysis. Advances in Water Science, 2013,24 (2) : 296-302. [程亮, 金菊良, 畉建强等. 干旱频率分析研究进展. 水科学进展, 2013, 24(2) : 296-302.]

[ 5 ] Xiao MZ, Zhang Q, Chen YQ et al. Hydrological drought frequency analysis of east river basin based on trivariate Copulas function. Journal of Natural Disasters, 2013, 22(2): 99-108. [肖名忠, 张强, 陈永勤等. 基于三变量 Copula 函数的 东江流域水文干旱频率分析. 自然灾害学报, 2013, 22(2): 99-108.]

[6] Chen YD, Zhang Q, Xiao M et al. Evaluation of risk of hydrological droughts by the trivariate Plackett copula in the East River basin (China). Natural Hazards, 2013, 68(2) : 529-547. DOI : 10.1007/s11069-013-0628-8.

[ 7 ] Salvadori G, Michele CD, Durante F. On the return period and design in a multivariate framework. Hydrology and Earth System Science, 2011, 15(11): 3293-3305. DOI: 10.5194/hess-15-3293-2011.

[ 8 ] Huang Q, Chen ZS. Multivariate flood risk assessment based on the secondary return period. J Lake Sci, 2015, 27 (2) : 352-360. DOI: 10.18307/2015.0221. [黄强, 陈子樧. 基于二次重现期的多变量洪水风险评估. 湖泊科学, 2015, 27 (2) : 352-360.]

[ 9 ] Li TY, Guo SL, Yan BW et al. Derivative design flood hydrograph based on trivariate joint distribution. Journal of Hydroelectric Engineering, 2013, 32(3): 10-14, 38. [李天元, 郭生练, 间宝伟等. 基于多变量联合分布推求设计洪水过 程线的新方法. 水力发电学报, 2013, 32(3): 10-14, 38.]

[10] Tu XJ, Chen XH, Zhao Y et al. Responses of hydrological drought properties and water shortage under changing environments in Dongjiang River basin. Advances in Water Science, 2016, 27(6):810-821. [涂新军, 陈晓宏, 赵勇等. 变化环 境下东江流域水文干旱特征及缺水响应. 水科学进展, 2016, 27(6): 810-821.]

[11] Yin ZJ, Huang W, Chen J. Impacts of reservoir flow regulation on hydrological drought. Journal of China Hydrology, 2009, 29(2) : 41-44. [尹正杰, 黄薇, 陈进. 水库径流调节对水文干旱的影响分析. 水文, 2009, 29(2) : 41-44.]

[12] Ma M, Song S, Ren L et al. Multivariate drought characteristics using trivariate Gaussian and Student t copulas. Hydrological Processes, 27(8) : 1175-1190. DOI: 10.1002/hyp.8432.

[13] Dobric J, Schmid F. A goodness of fit test for copulas based on Rosenblatt's transformation. Computation Statistics and Data Analysis, 2007, 51(9) : 4633-4642. DOI: 10.1016/j.csda.2006.08.012.

[14] Tu X, Singh VP, Chen X et al. Uncertainty and variability in bivariate modeling of hydrological droughts. Stochastic Environmental Research and Risk Assessment, 2016, 30(5) : 1317-1334. DOI : 10.1007/s00477-015-1185-3.

[15] Tu XJ, Du YL, Chen XH et al. Modeling and design on joint distribution of precipitation and tide in the coastal city. Advances in Water Science, 2017, 28(1) : 49-58. [涂新军, 杜奕良, 陈晓宏等. 滨海城市雨潮遭遇联合分布模拟与设 计. 水科学进展, 2017, 28(1): 49-58.]

[16] Tu XJ, Chen XH. Characteristics variability study of regional river runoff time series based on change point recognition. Journal of Natural Resources, 2010, 25(11) : 1930-1937. DOI: 10.11849/zrzyxb.2010.11.012. [涂新军, 陈晓宏. 基于 变点识别的区域河川径流量特征值变异研究. 自然资源学报, 2010, 25(11)：1930-1937.]

[17] Tu XJ, Chen XH, Zhang Q et al. Streamflow annual distribution and its influencing factors in Dongjiang River, South China. Advances in Water Science, 2012, 23(4) : 493-501. DOI: 32.1309.P.20120614.2158.007. [涂新军, 陈晓宏, 张强 等. 东江径流年内分配特征及影响因素贡献分解. 水科学进展, 2012, 23(4) : 493-501.] 